\title{
Kerr black holes with synchronised scalar hair and boson stars in the Einstein-Friedberg-Lee-Sirlin model
}

\author{
J. Kunz, ${ }^{a}$ I. Perapechka ${ }^{b}$ and Ya. Shnir ${ }^{c, d}$ \\ ${ }^{a}$ Institut für Physik, Universität Oldenburg, \\ Postfach 2503 D-26111 Oldenburg, Germany \\ ${ }^{b}$ Department of Theoretical Physics and Astrophysics, Belarusian State University, \\ Nezavisimosti Avenue 4, Minsk 220004, Belarus \\ ${ }^{c}$ BLTP, JINR, \\ Dubna 141980, Moscow Region, Russia \\ ${ }^{d}$ Department of Theoretical Physics, Tomsk State Pedagogical University, \\ Russia \\ E-mail: jutta.kunz@uni-oldenburg.de, jonahex111@outlook.com, \\ shnir@theor.jinr.ru
}

AbStRaCt: We consider the Friedberg-Lee-Sirlin model minimally coupled to Einstein gravity in four spacetime dimensions. The renormalizable Friedberg-Lee-Sirlin model consists of two interacting scalar fields, where the mass of the complex scalar field results from the interaction with the real scalar field which has a finite vacuum expectation value. We here study a new family of self-gravitating axially-symmetric, rotating boson stars in this model. In the flat space limit these boson stars tend to the corresponding Q-balls. Subject to the usual synchronization condition, the model admits spinning hairy black hole solutions with two different types of scalar hair. We here investigate parity-even and parity-odd boson stars and their associated hairy black holes. We explore the domain of existence of the solutions and address some of their physical properties. The solutions exhibit close similarity to the corresponding boson stars and Kerr black holes with synchronised scalar hair in the $O(3)$-sigma model coupled to Einstein gravity and to the corresponding solutions in the Einstein-Klein-Gordon theory with a complex scalar field, where the latter are recovered in a limit.

KEywords: Black Holes, Classical Theories of Gravity, Solitons Monopoles and Instantons

ArXiv EPrint: 1904.13379 


\section{Contents}

1 Introduction 1

2 The model 3

3 Spinning axially-symmetric configurations 5

3.1 Stationary axially symmetric ansatz and boundary conditions 5

$\begin{array}{ll}3.2 & \text { Quantities of interest and Smarr relation } \\ \end{array}$

4 Results $\quad 7$

4.1 Numerical implementation $\quad 7$

4.2 Stationary spinning self-gravitating solitons and black holes with synchronised hair

\section{Introduction}

The investigation of self-gravitating scalar field configurations in $3+1$ dimensional asymptotically flat spacetime has attracted much interest in the last decades. One of the reasons is that a fundamental scalar field is a necessary ingredient in models of inflation. Thus such fields might play an important role in the evolution of the early Universe. On the other hand, when scalar fields are present in the Universe, the gravitational interaction may lead to gravitational collapse and form localized gravitating objects. In the case of a complex scalar field so-called boson stars might arise, that is, compact, stationary configurations where the scalar field possesses a harmonic time dependence $[1,2]$.

Similar static localized field configurations with finite energy exist in Einstein-Skyrme theory [3-5] and in SU(2) Einstein-Yang-Mills theory [6] or Einstein-Yang-Mills-Higgs theory [7-10]. Certain types of localized gravitating solutions, like boson stars with appropriate interactions, or gravitating monopoles, sphalerons and Skyrmions, are linked to the corresponding flat space solutions, which represent topological solitons/Q-balls [11, 12], or monopoles [13, 14], sphalerons [15], and Skyrmions [16], respectively.

In particular, the Friedberg-Lee-Sirlin model [11] provides an interesting example of a simple renormalizable two-component scalar field theory with natural interaction terms. In this model the complex scalar becomes massive due to the coupling with the real scalar field, since the latter has a finite vacuum expection value generated via a symmetry breaking potential. The Q-ball solutions of this model then appear because of the phase rotation of the complex scalar field, and the coupling to gravity leads to the respective boson stars.

Gravitating localized solutions of another type are bound by gravity. Examples are boson stars without appropriate self-interactions [1, 2], or the Bartnik-McKinnon solutions [6]. These do not possess a flat space limit. 
All these self-gravitating configurations exist for a certain range of values of the parameters of the respective theory, For instance, there are two branches of self-gravitating Skyrmions [4, 5], where the lower in energy branch is linked to the flat space Skyrmion in the limit of a vanishing effective gravitational coupling. This lower branch of solutions then ends at some critical maximal value of the gravitational coupling, where it bifurcates with the second, higher in energy branch, which extends all the way backwards to the limit of zero coupling. Likewise, for gravitating monopoles the lower in energy branch is linked to the flat space monopole, however, the second branch ends when an extremal Reissner-Nordström configuration is reached (in the exterior) [7, 8].

Boson star configurations, on the other hand, possess a presumably infinite number of branches, representing an inspiraling of the solutions towards a limiting solution, when the dependence of the mass or particle number on the frequency or on the radius is considered [17-20]. This spiraling behavior is reminiscent of the mass radius relation of neutron stars beyond the maximum mass star. For other physical quantities this translates into an oscillating behavior for boson stars and neutron stars, alike.

Notably, many of those regular particle-like gravitating solitons, like the gravitating monopoles, sphalerons and Skyrmions or the Bartnik-McKinnon solutions, can be linked to hairy black holes in the limit of vanishing event horizon radius [3, 21]. These static black holes provide the first known counter-examples to the celebrated no-hair conjecture [22] (see also [23-25] for further references and discussion).

In contrast, the spherically symmetric boson star solutions $[1,2,17,18]$ cannot be generalized to contain a small Schwarzschild black hole in their inner region. More general, it has been shown that there is no regular static asymptotically flat solution with an event horizon in these models with a complex scalar field, which harmonically depends on time $[26,27]$. This situation is not unique, however. For example, regular static spherically symmetric self-gravitating solutions of the generalized Skyrme model [28, 29] terminate at a singular solution, and cannot be continuously connected to a static hairy black hole [30].

An interesting aspect for all such gravitating regular and black hole solutions is their possible generalization to include rotation, since rotation is ubiquitous in the Universe. For instance, for boson stars there is no slow rotating limit [31], however, they can rotate rapidly [32-34]. Other rotating regular configurations include the gravitating Skyrmions and $Q$-clouds [35], gravitating dyons and vortex rings [36, 37] or the spinning topological solitons of the non-linear $\mathrm{O}(3)$ sigma model [38].

Whereas various rotating hairy black holes were obtained before [39-44], only recently a spinning complex scalar field was considered in a Kerr black hole spacetime, first perturbatively and then with back reaction [45-52]. In that case, the rotating hairy black holes obey a synchronization condition between the angular velocity of the event horizon and a phase frequency of the scalar field. Numerous hairy black holes obeying such a synchronization condition have been studied by now. Further examples are given in [38, 53-63].

Interestingly, the Q-balls in the Friedberg-Lee-Sirlin model in flat space may also exist in the limiting case of vanishing scalar potential $[64,65]$. In this limit the real component of the scalar field becomes massless, thus it possesses a Coulomb-like asymptotic tail. This leads to the interesting question, as to whether one can find similar rotating self-gravitating 
asymptotically flat solutions, which are either regular or possess an event horizon. Such black hole solutions would represent a new type of hairy black holes, quite different from the scalarized hairy black holes of scalar-tensor theory, where the real scalar field is associated with the gravitational interaction [53].

In this paper we answer this question positively. In particular, we extend the study of the spinning boson stars and hairy black holes, by constructing new families of stationary rotating solutions in the Friedberg-Lee-Sirlin model minimally coupled to Einstein gravity. The boson star solutions possess properties that are similar to those of the rotating boson stars with a single complex scalar field [32-34], featuring both parity-even and parity-odd configurations $[19,20]$. In the flat space limit, they are linked to the corresponding spinning flat-space Q-balls [19, 20, 65-68].

All these rotating boson star solutions are related to rotating hairy black hole solutions, where the phase rotation of the massive complex component is synchronized with the angular velocity of the horizon. An important novelty of these solutions is the fact that in the limit of vanishing potential these solutions correspond to black holes with two different types of scalar hair, where the second real scalar field is massless. Further, in the opposite limit of infinite mass of the real component, the solutions of the model effectively reduce to the corresponding boson stars and hairy black holes of the Einstein-Klein-Gordon model $[24,46,48,63]$. We here show that, depending on the values of the parameters of the model and, in particular, the horizon radius parameter, the branch structure of the solutions varies from the typical spiral pattern of boson stars [17-20, 69, 70], to a simpler branch structure known for various types of hairy black holes $[4,5,28,29,38-43,46-$ $48,53,53-57,60-63,71]$.

\section{The model}

We consider the $3+1$ dimensional action

$$
S=\int d^{4} x \sqrt{-g}\left(\frac{R}{4 \alpha^{2}}-\mathcal{L}_{m}\right)
$$

where the gravity part is the usual Einstein-Hilbert action, $\alpha^{2}=4 \pi G$ is the gravitational coupling, $R$ is the curvature scalar and $G$ is Newton's constant. The Lagrangian of the matter fields $\mathcal{L}_{m}$ is given by the two-component Friedberg-Lee-Sirlin model [11]

$$
\mathcal{L}_{\mathrm{m}}=\frac{1}{2}\left(\partial_{\mu} \psi\right)^{2}+\left|\partial_{\mu} \phi\right|^{2}+m^{2} \psi^{2}|\phi|^{2}-\mu^{2}\left(\psi^{2}-v^{2}\right)^{2} .
$$

Here a real self-interacting scalar field $\psi$ is coupled to a complex scalar field $\phi$, the parameters $m$ and $\mu$ are the real positive coupling constants and $v$ is the vacuum expectation value of the real scalar field $\psi$. The first two parts in (2.2) are the usual kinetic terms for the real and complex field, respectively, the third is the interaction term, and the last term gives the potential of the real scalar field.

The potential is chosen such that in the vacuum $\psi \rightarrow v$, and the complex field $\phi$ becomes massive with mass $m v$ due to the coupling with its real partner. When expanded 
around its vacuum expectation value $v$, the fluctuations of the real scalar field are associated with a mass $\sqrt{8} \mu v$, thus $\mu$ represents a mass parameter for the real scalar field. In the limit of vanishing mass parameter $\mu \rightarrow 0$ but fixed vacuum expectation value $v$, the real scalar field becomes massless and thus long-ranged. The complex component $\phi$ still acquires mass in this limit due to the coupling with the Coulomb-like field $\psi$.

Note that two of the four parameters of the model (2.1) can be rescaled away via transformations of the coordinates and the fields,

$$
\alpha \rightarrow v \alpha, \quad x_{\mu} \rightarrow m v x_{\mu}, \quad \psi \rightarrow \frac{\psi}{v}, \quad \phi \rightarrow \frac{\phi}{v} .
$$

After such a rescaling $\tilde{\alpha}=v \alpha$ and $\tilde{\mu}=\mu / m$ will be the remaining parameters. However, we will only set $v=1$ in the following, retaining $\tilde{\alpha}$ (omitting the tilde), $m$ and $\mu$.

The model (2.1) is invariant under global U(1) transformations of the complex field $\phi \rightarrow \phi e^{\delta}$, where the parameter $\delta$ is a constant. The following Noether current is associated with this symmetry,

$$
j_{\mu}=i\left(\phi \partial_{\mu} \phi^{*}-\phi^{*} \partial_{\mu} \phi\right),
$$

with the corresponding charge $Q=\int \sqrt{-g} j^{t} d^{3} x$.

Variation of the action (2.1) with respect to the metric leads to the Einstein equations

$$
R_{\mu \nu}-\frac{1}{2} R g_{\mu \nu}=2 \alpha^{2} T_{\mu \nu}
$$

where

$$
T_{\mu \nu}=\partial_{\mu} \psi \partial_{\nu} \psi+\left(\partial_{\mu} \phi \partial_{\nu} \phi^{*}+\partial_{\nu} \phi \partial_{\mu} \phi^{*}\right)-\mathcal{L}_{\mathrm{m}} g_{\mu \nu}
$$

is the stress-energy tensor of the scalar fields.

The corresponding equations of motion of the scalar fields read

$$
\begin{aligned}
\square \psi & =2 \psi\left(m^{2}|\phi|^{2}+2 \mu^{2}\left(1-\psi^{2}\right)\right), \\
\square \phi & =m^{2} \psi^{2} \phi,
\end{aligned}
$$

where $\square$ represents the covariant d'Alembert operator. It follows from the linearized field equations (2.7) that the parameters $\mu$ and $m$ indeed determine the mass of the real and complex scalar fields, respectively. Notably, the flat-space localized regular solutions of the Friedberg-Lee-Sirlin model (2.2) exist in the limit of vanishing scalar potential, $\mu \rightarrow 0$, when the vacuum expectation value of the real component $\psi$ is kept non-zero [64,65]. They represent Q-balls with a long-range massless scalar component. These Q-balls are similar to those of the Wick-Cutkosky model [72, 73] revisited recently in [74, 75].

In the opposite limit, $\mu \rightarrow \infty$, the real component of the model (2.2) trivializes, $\psi=1$, and the massive complex field $\phi$ satisfies the Klein-Gordon equation. Clearly, spatially localized stationary spinning solutions of this equation do not exist in the flat space. However, there are families of corresponding boson stars and hairy black holes with synchronised hair in the complex-Klein-Gordon field theory minimally coupled to Einstein's gravity $[24,46,48,61,63]$. 


\section{Spinning axially-symmetric configurations}

\subsection{Stationary axially symmetric ansatz and boundary conditions}

Spherically symmetric self-gravitating regular solutions of the equations (2.5), (2.7) do not admit spherically symmetric generalizations with a horizon [26]. In the present paper we shall consider spinning regular and hairy black hole solutions to the system (2.5), (2.7). We note, that spinning self-gravitating regular solutions of an extended model with two complex interacting scalar fields were considered before [76].

To obtain stationary spinning axially-symmetric solutions we take into account the presence of two commuting Killing vector fields $\xi=\partial_{t}$ and $\eta=\partial_{\varphi}$, where $t$ and $\varphi$ are the time and azimuthal coordinates, respectively. In these coordinates the metric can be written in isotropic coordinates in the Lewis-Papapetrou form

$$
d s^{2}=-F_{0} d t^{2}+F_{1}\left(d r^{2}+r^{2} d \theta^{2}\right)+r^{2} \sin ^{2} \theta F_{2}\left(d \varphi-\frac{W}{r} d t\right)^{2},
$$

where the four metric functions $F_{0}, F_{1}, F_{2}$ and $W$ depend on $r$ and $\theta$ only.

For the scalar fields we adopt the axially-symmetric ansatz

$$
\psi=X(r, \theta), \quad \phi=Y(r, \theta) e^{i(\omega t+n \varphi)},
$$

where the real profile functions $X$ and $Y$ depend on the radial coordinate $r$ and the polar angle $\theta$, the frequency of the spinning complex field $\omega$ is a parameter of the model, and $n \in \mathbb{Z}$ is the azimuthal winding number, also referred to as rotational quantum number. For stationary spherically symmetric configurations $n=0$, and the system of equations (2.5), (2.7) reduces to the set of equations of the corresponding boson stars.

To obtain hairy black holes, we assume the existence of a rotating event horizon, located at a constant value of the radial variable, $r=r_{h}>0$. The Killing vector of the horizon is the helicoidal vector field

$$
\chi=\xi+\Omega_{h} \eta,
$$

where the horizon angular velocity $\Omega$ is fixed by the value of the metric function $W$ on the horizon

$$
\Omega_{h}=-\left.\frac{g_{\phi t}}{g_{t t}}\right|_{r=r_{h}}=\left.W\right|_{r=r_{h}} .
$$

The presence of a rotating horizon allows to form stationary scalar clouds, supported by the synchronisation condition $[24,38,45,46,48]$

$$
\omega=n \Omega_{h}
$$

between the event horizon angular velocity $\Omega_{h}$, the complex scalar field frequency $\omega$, and the winding number $n$. This condition implies that there is no flux of the complex scalar field into the black hole. 
It is convenient to make use of the exponential parametrization of the metric fields

$$
F_{0}=\frac{\left(1-\frac{r_{h}}{r}\right)^{2}}{\left(1+\frac{r_{h}}{r}\right)^{2}} e^{f_{0}}, \quad F_{1}=\left(1+\frac{r_{h}}{r}\right)^{4} e^{f_{1}}, \quad F_{2}=\left(1+\frac{r_{h}}{r}\right)^{4} e^{f_{2}},
$$

where the functions $f_{i}$ depend on the radial coordinate $r$ and the polar angle $\theta$. Then a power series expansion near the horizon yields the following conditions of regularity for the profile functions $X, Y$ and the metric functions $f_{i}$ :

$$
\left.\partial_{r} X\right|_{r=r_{h}}=\left.\partial_{r} Y\right|_{r=r_{h}}=\left.\partial_{r} f_{0}\right|_{r=r_{h}}=\left.\partial_{r} f_{1}\right|_{r=r_{h}}=\left.\partial_{r} f_{2}\right|_{r=r_{h}}=0 .
$$

These Neumann boundary conditions must be supplemented by the synchronization condition (3.4) imposed on the metric function $W$.

Requirement of asymptotic flatness implies that, as $r \rightarrow \infty$, the metric approaches the Minkowski limit, and the scalar fields are taking their vacuum values

$$
\left.X\right|_{r \rightarrow \infty}=1,\left.\quad Y\right|_{r \rightarrow \infty}=\left.f_{0}\right|_{r \rightarrow \infty}=\left.f_{1}\right|_{r \rightarrow \infty}=\left.f_{2}\right|_{r \rightarrow \infty}=\left.W\right|_{r \rightarrow \infty}=0 .
$$

Demanding axial symmetry and regularity imposes the following boundary conditions on the symmetry axis for $\theta=0, \pi$

$$
\left.\partial_{\theta} X\right|_{\theta=0, \pi}=\left.Y\right|_{\theta=0, \pi}=\left.\partial_{\theta} f_{0}\right|_{\theta=0, \pi}=\left.\partial_{\theta} f_{1}\right|_{\theta=0, \pi}=\left.\partial_{\theta} f_{2}\right|_{\theta=0, \pi}=\left.\partial_{\theta} W\right|_{\theta=0, \pi}=0 .
$$

We also require the solutions to be $\mathbb{Z}_{2}$-symmetric with respect to reflection symmetry $\theta \rightarrow \pi-\theta$ in the equatorial plane $\theta=\pi / 2$. Thus, we can restrict the range of values of the angular variable as $\theta \in[0, \pi / 2]$. The corresponding boundary conditions on the equatorial plane are

$$
\left.\partial_{\theta} X\right|_{\theta=\frac{\pi}{2}}=\left.\partial_{\theta} f_{0}\right|_{\theta=\frac{\pi}{2}}=\left.\partial_{\theta} f_{1}\right|_{\theta=\frac{\pi}{2}}=\left.\partial_{\theta} f_{2}\right|_{\theta=\frac{\pi}{2}}=\left.\partial_{\theta} W\right|_{\theta=\frac{\pi}{2}}=0 .
$$

Further, there are two different types of axially symmetric solutions, which possess different parity of the complex scalar field [19, 20,65]. The corresponding boundary conditions on the complex component of the field in the equatorial plane are $\left.\partial_{\theta} Y\right|_{\theta=\frac{\pi}{2}}=0$ for parity-even solutions, and $\left.Y\right|_{\theta=\frac{\pi}{2}}=0$ for parity-odd configurations.

Note that the absence of a conical singularity on the symmetry axis requires that the deficit angle should vanish, $\delta=2 \pi\left(1-\lim _{\theta \rightarrow 0} \frac{F_{2}}{F_{1}}\right)=0$. Hence the solutions should satisfy the constraint $\left.F_{2}\right|_{\theta=0}=\left.F_{1}\right|_{\theta=0}$. In our numerical scheme we explicitly checked this condition on the symmetry axis.

\subsection{Quantities of interest and Smarr relation}

Asymptotic expansions of the metric functions at the horizon and at spatial infinity yield a number of physical observables. The total ADM mass $M$ and the angular momentum $J$ of the spinning hairy black holes can be read off from the asymptotic subleading behaviour of the metric functions as $r \rightarrow \infty$

$$
g_{t t}=-1+\frac{\alpha^{2} M}{\pi r}+O\left(\frac{1}{r^{2}}\right), \quad g_{\varphi t}=\frac{\alpha^{2} J}{\pi r} \sin ^{2} \theta+O\left(\frac{1}{r^{2}}\right) .
$$


The ADM charges can be represented as sums of the contributions from the event horizon and the scalar hair, $M=M_{h}+M_{\Phi}$ and $J=J_{h}+J_{\Phi}$, respectively. These contributions can be evaluated separately via Komar integrals

$$
\begin{aligned}
M_{h} & =-\frac{1}{2 \alpha^{2}} \oint_{\mathrm{S}} d S_{\mu \nu} \nabla^{\mu} \xi^{\nu}, & J_{h} & =\frac{1}{4 \alpha^{2}} \oint_{\mathrm{S}} d S_{\mu \nu} \nabla^{\mu} \eta^{\nu}, \\
M_{\Phi} & =-\frac{1}{\alpha^{2}} \int_{V} d S_{\mu}\left(2 T_{\nu}^{\mu} \xi^{\nu}-T \xi^{\mu}\right), & J_{\Phi} & =\frac{1}{2 \alpha^{2}} \int_{\mathrm{V}} d S_{\mu}\left(T_{\nu}^{\mu} \eta^{\nu}-\frac{1}{2} T \eta^{\mu}\right),
\end{aligned}
$$

where $S$ is the horizon 2-sphere and $V$ denotes an asymptotically flat spacelike hypersurface bounded by the horizon.

Analogously to other axially symmetric stationary rotating boson stars [19, 20], selfgravitating solitons of the non-linear sigma model [38] and vortons [76], one obtains the quantization relation for the angular momentum of the spinning complex scalar component of the model, $J_{\Phi}=n Q$, where $Q$ is its Noether charge and $n$ its winding number.

The physically interesting horizon properties include the Hawking temperature $T_{h}$, which is proportional to the surface gravity $\kappa^{2}=-\frac{1}{2} \nabla_{\mu} \chi_{\nu} \nabla^{\mu} \chi^{\nu}$

$$
T_{h}=\frac{\kappa}{2 \pi}=\frac{1}{16 \pi r_{h}} \exp \left[\left.\left(f_{0}-f_{1}\right)\right|_{r=r_{h}}\right]
$$

where $\chi$ is the horizon Killing vector (3.3). Of interest is also the horizon area $A_{h}$, which is given by

$$
A_{h}=32 \pi r_{h}^{2} \int_{0}^{\pi} d \theta \sin \theta \exp \left[\left.\left(f_{1}+f_{2}\right)\right|_{r=r_{h}}\right] .
$$

The observables are related via the Smarr relation

$$
M=2 T_{h} S+2 \Omega_{h} J_{h}+M_{\Phi},
$$

where $S=\frac{\pi}{\alpha^{2}} A_{h}$ is the entropy of the black hole and $M_{\Phi}$ is the energy of the scalar fields outside the event horizon (3.11). Another relation between the physical quantities of the hairy black hole is the first law of thermodynamics

$$
d M=T_{h} d S+\Omega_{h} d J .
$$

\section{Results}

\subsection{Numerical implementation}

The set of six coupled non-linear elliptic partial differential equations of the functions $X, Y, W, f_{0}, f_{1}, f_{2}$, which parametrize the system (2.5), (2.7), has been solved numerically subject to the boundary conditions (3.6)-(3.8). We have made use of a fourth-order finite differences scheme, where the system of equations is discretized on a grid with $101 \times 101$ points. To facilitate the calculations in the near horizon area, we have introduced the new radial coordinate $x=\frac{r-r_{h}}{r+c}$, which maps the semi-infinite region $[0, \infty)$ onto the unit interval $[0,1]$. Here $c$ is an arbitrary constant used to adjust the contraction of the grid. The emerging system of nonlinear algebraic equations is solved using a modified Newton 
method. The underlying linear system is solved with the Intel MKL PARDISO sparse direct solver. The errors are on the order of $10^{-5}$. All calculation have been performed using CESDSOL ${ }^{1}$ library.

\subsection{Stationary spinning self-gravitating solitons and black holes with synchro- nised hair}

When constructing spinning gravitating solutions, we start with the corresponding flat spacetime configurations [65] as initial guesses. Since the present system possesses a large space of parameters, we restrict our analysis to two particular values of the mass parameter $\mu, \mu^{2}=0.25$ and $\mu=0$ (i.e., the limiting case of vanishing potential), recall that the mass $m$ of the complex field can be rescaled to $m=1$. The regular self-gravitating solitonic solutions are found by replacing the boundary conditions at the horizon $r_{h}$ with appropriate boundary conditions at $r=0$.

As usual, the stationary spinning solutions exist within a restricted interval of the scaled frequency $\omega \in\left[\omega_{\min }, \omega_{\max }=1\right]$. The upper bound of the frequency $\omega$ corresponds to the mass of the complex scalar field, the lower critical frequency depends on the value of the second mass parameter $\mu$. As $\omega \rightarrow \omega_{\max }=1$ the spinning configurations smoothly approach linearized perturbations around Minkowski spacetime, with the ADM mass $M$ tending to zero.

Note that, unlike the corresponding solutions of the non-renormalizable flat space model with a single complex field and a sextic potential $[19,20]$, there is no lower bound on the frequency in the flat space limit of the present model: the Friedberg-Lee-Sirlin Qballs exist for all non-zero values of the frequency $\omega<m[64,65]$. As we shall see, the coupling to gravity changes this, and a lower critical value of the frequency appears.

Setting the winding number $n$ to $n=0$ reduces the above system to the spherically symmetric case of the regular boson star solutions. In figure 1 we display the ADM mass $M$ as a function of rescaled frequency (left) and the mass $M$ vs the charge $Q$ for oscillating $n=0$ parity-even boson stars in the model (2.1).

Notably, the coupling to gravity reduces the interval of values of the frequency $\omega$, and the spherically symmetric boson stars exist in a more limited frequency range. The minimal value $\omega_{\min }>0$ depends on the strength of the gravitational coupling $\alpha$ and on the value of the mass parameter $\mu$, see figure 1, left plot. As expected, these boson stars exhibit a typical spiral-like frequency dependence of the charge and the mass, and should approach finite limiting values at the centers of the spirals.

Considering the $n=0$ configurations we do not find spherically symmetric hairy black holes, as expected. However, taking $n \geq 1$, we obtain both boson stars with non-zero angular momentum, and spinning hairy black holes. Furthermore, for $n \geq 1$ there are two different types of spinning configurations, [19, 20,61, 63, 66], referred to as parity-even and parity-odd solutions, respectively.

\footnotetext{
${ }^{1}$ Complex Equations - Simple Domain partial differential equations SOLver is a $\mathrm{C}++$ library developed by IP. It provides tools for the discretization of an arbitrary number of arbitrarily nonlinear equations with arbitrary boundary conditions on direct product arbitrary dimensional grids with arbitrary order of accuracy.
} 

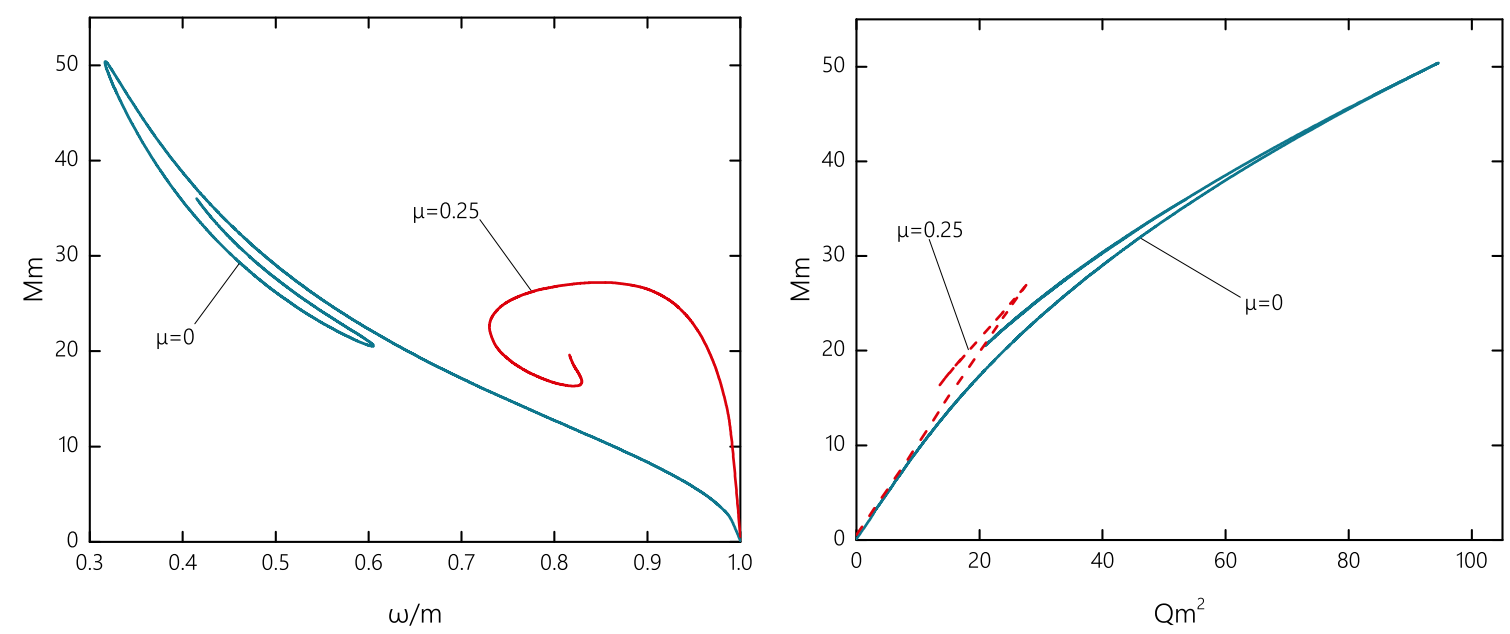

Figure 1. The ADM mass $M$ vs the angular frequency $\omega$ (left plot) and Noether charge $Q$ (right plot) at $\mu / m=0.25, \mu=0$ and $\alpha=0.5$ for parity-even $n=0$ boson stars.

In figure 2 the $\mathrm{ADM}$ mass $M$ of the $n=1$ parity-even and parity-odd solutions is exhibited versus the frequency $\omega$ for a set of values of the horizon radius parameter $r_{h}$, including the regular limit $r_{h}=0$, for the chosen values of the mass parameter $\mu^{2}=0.25$ and the gravitational coupling $\alpha=0.5$. The families of boson star solutions are found in the $r_{h} \rightarrow 0$ limit. They emerge from the vacuum at $\omega_{\max }=1$, and form the fundamental branch of solutions. Both the dependence of the mass and the Noether charge of the regular configurations on the frequency form an inspiraling pattern, which is typical for spinning boson star solutions and some other types of gravitating solitons [19, 20, 38, 53, 76].

As the fundamental branch of regular solutions arises from the upper limiting frequency value $\omega_{\max }$, the mass $M$ of the configurations gradually increases with decreasing $\omega$ and, for all values of the mass parameter $\mu$ not too close to zero, the dependence $M(\omega)$ possesses a maximum at some critical value of the frequency $\omega_{\mathrm{M}}$. As the frequency decreases below that point, the mass of the solutions decreases until the minimum frequency $\omega_{\min }$ is reached. Here the fundamental forward branch merges with a second (backward) branch, leading to a counterclockwise inspiraling of the mass curve $M(\omega)$.

In general, for the same set of values of the parameters of the model, the mass of the parity-odd configurations is considerably higher than the mass of the parity-even boson stars, as seen in figure 2, left column. The parity-odd $n=1$ solutions represent a new family of boson stars, which in the flat space limit are linked to the corresponding Q-balls [65].

We have found that black holes with synchronized hair exist in a small interval of the event horizon radius parameter, typically $r_{h}<0.07$, see figure 2 . For very small values of the horizon radius $r_{h}, r_{h}<0.02$, the inspiraling critical behavior is changed to a multibranch structure with a few branches only, leading toward a second upper critical value of the frequency $\omega_{\mathrm{cr}}^{(1)}<1$, see figure 2 , upper plots. In this limit, the real scalar component trivializes and the lower branch ends on the vacuum Kerr black holes with stationary scalar Klein-Gordon clouds, see [24, 38, 45, 46, 48, 54]. 

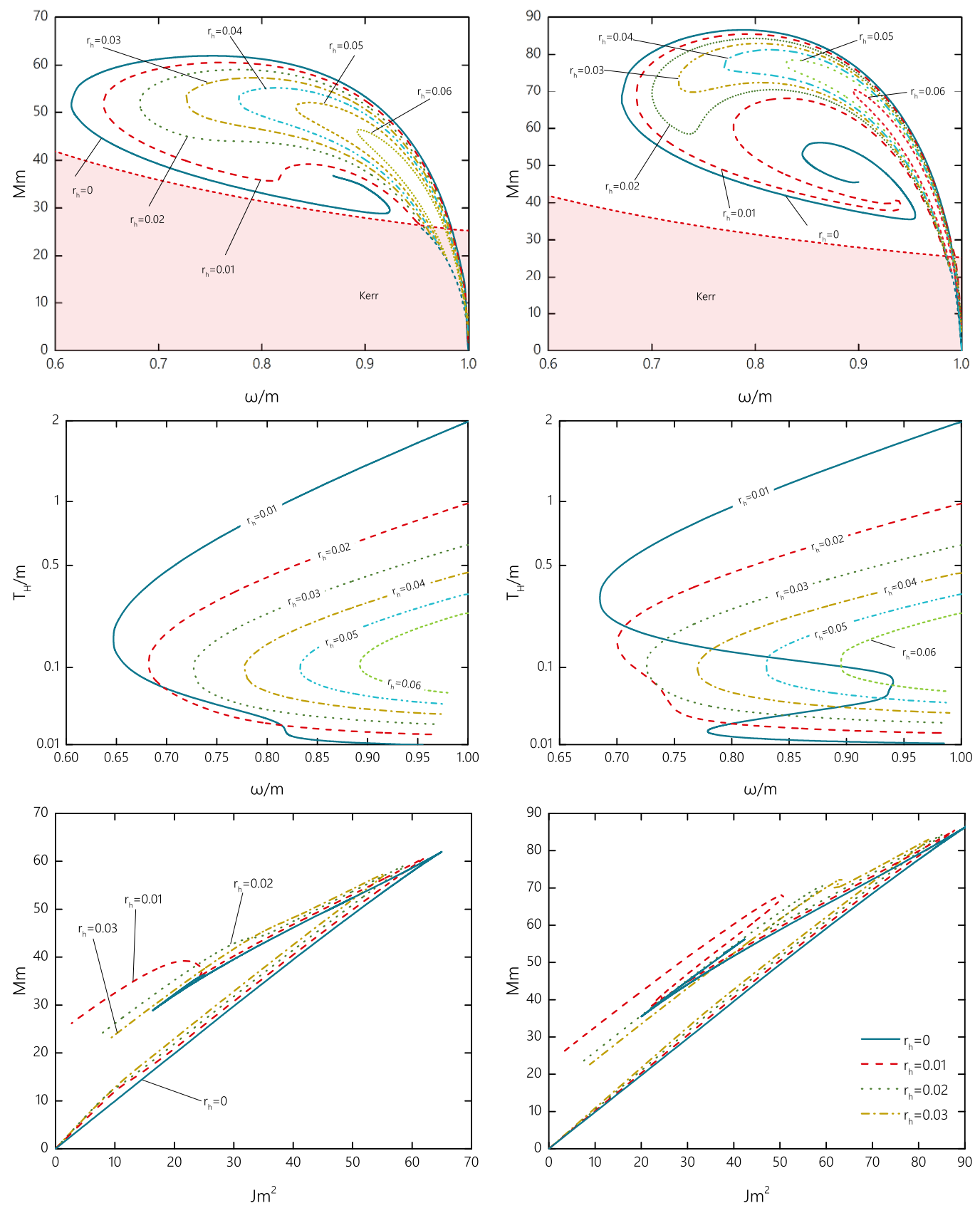

Figure 2. The ADM mass $M$ (upper row) and the Hawking temperature $T_{h}$ (middle row) vs the frequency $\omega$, and the mass $M$ vs the angular momentum $J$ (bottom row) for a set of values of the horizon radius parameter $r_{h}$ for $n=1$ rotating parity-even (left column) and parity-odd (right column) hairy black holes at $\mu / m=0.25$ and $\alpha=0.5$. In the upper plots, here, and in the subsequent figures below, the shaded area corresponds to the domain of existence of vacuum Kerr black holes, the red short-dashed line to the extremal vacuum Kerr black holes, and the blue short-dashed line to the subset of vacuum Kerr black holes with stationary scalar Klein-Gordon clouds. 
As the horizon radius $r_{h}$ increases, the multibranch structure is replaced by a twobranch scenario with the second (lower, backward) branch ending on the limiting Kerr solution as $\omega \rightarrow \omega_{\mathrm{cr}}^{(1)}$, see figure 2 , left column. The maximum value of the frequency along the second branch $\omega_{\mathrm{cr}}^{(1)}$ slowly increases as the horizon radius $r_{h}$ grows, and approaches $\omega_{\max }=1$ at some maximal value of the horizon radius $r_{h}$ as the loop shrinks to zero. The branches exist up to the limit $\alpha=0$, where the regular solutions approach the corresponding flat space Q-ball configurations while the solutions with non-zero horizon radius $r_{h}$ become linked to the scalar clouds spinning around the Kerr black hole in the probe limit.

The Hawking temperature constantly decreases as $\omega$ varies along the branches, see figure 2, middle row. Note that the configurations with the smallest horizon radius $r_{h}$ have the smallest temperature, both for the parity-even and parity-odd solutions.

Figure 2 also presents the ADM mass as a function of the angular momentum $J$, see the lower column. The mass of the regular spinning configurations with horizon radius $r_{h}=0$ exhibits a typical zig-zag behavior known for boson stars [17-20, 53, 68, 70]. As the horizon radius increases, it becomes replaced with the two-branch pattern, which is well known for the Kerr scalar clouds and other similar solutions [24, 38, 45, 46, 48, 54]. Here the lower branch corresponds to the values of the frequency smaller than $\omega_{\mathrm{cr}}^{(1)}$, while the upper branch represents the values $\omega>\omega_{\mathrm{cr}}^{(1)}$.

This pattern is similar to the corresponding branch structure of the usual boson stars [17-20] and spinning hairy black holes [38, 45-52]. The analysis of the stability of the solutions of that type [78-80] suggests that the appearance of a cusp in the mass vs charge dependence indicates a transition from a stable fundamental branch to an unstable branch. Moreover, the spinning boson stars become unstable, when they develop an ergoregion. Analogous arguments should hold also in the case of regular and hairy black hole solutions of the Einstein-Friedberg-Lee-Sirlin model.

In figure 3 we present the horizon values of the massive scalar fields $X\left(r_{h}\right)$ and $Y\left(r_{h}\right)$ as functions of the frequency $\omega$ for $\mu^{2}=0.25$. Both the parity-even and the parity-odd solutions start at $\omega_{\max }=1$ from the regular limits $X\left(\omega_{\max }\right)=1, Y\left(\omega_{\max }\right)=0$.

To conclude the analysis of the massive $\left(\mu^{2}=0.25\right)$ spinning configurations, we exhibit in figure 4 the dependence of the scalar mass of the $n=1$ parity-even solutions on the gravitational coupling $\alpha$ for some set of the values of the horizon radius $r_{h}$. One can see that the mass of the solutions on both branches decreases monotonically as $\alpha$ increases. In the limiting case $\alpha \rightarrow 0$ the solutions approach the Q-balls spinning on the Minkowski or Schwarzschild background.

The situation changes drastically for the spinning solutions when $\mu \rightarrow 0$. First, we observe that the spinning component of the coupled configuration falls off exponentially. It remains massive, whereas the real scalar field decays as $\sim r^{-1}$. Figure 5 exhibits the profile functions of both parity-even and parity-odd scalar field functions $X(r, \theta)$ and $Y(r, \theta)$ for the massive $\mu^{2}=0.25$ and the massless $(\mu=0)$ case. Thus, the massless limit provides a new type of hairy black hole with hair of two different types, that are short- and longranged, respectively.

In figure 6 we display the ADM mass $M$ (left plot) and the Hawking temperature $T_{H}$ (right plot) of the parity-even $n=1$ configurations versus the frequency $\omega$. Similar 

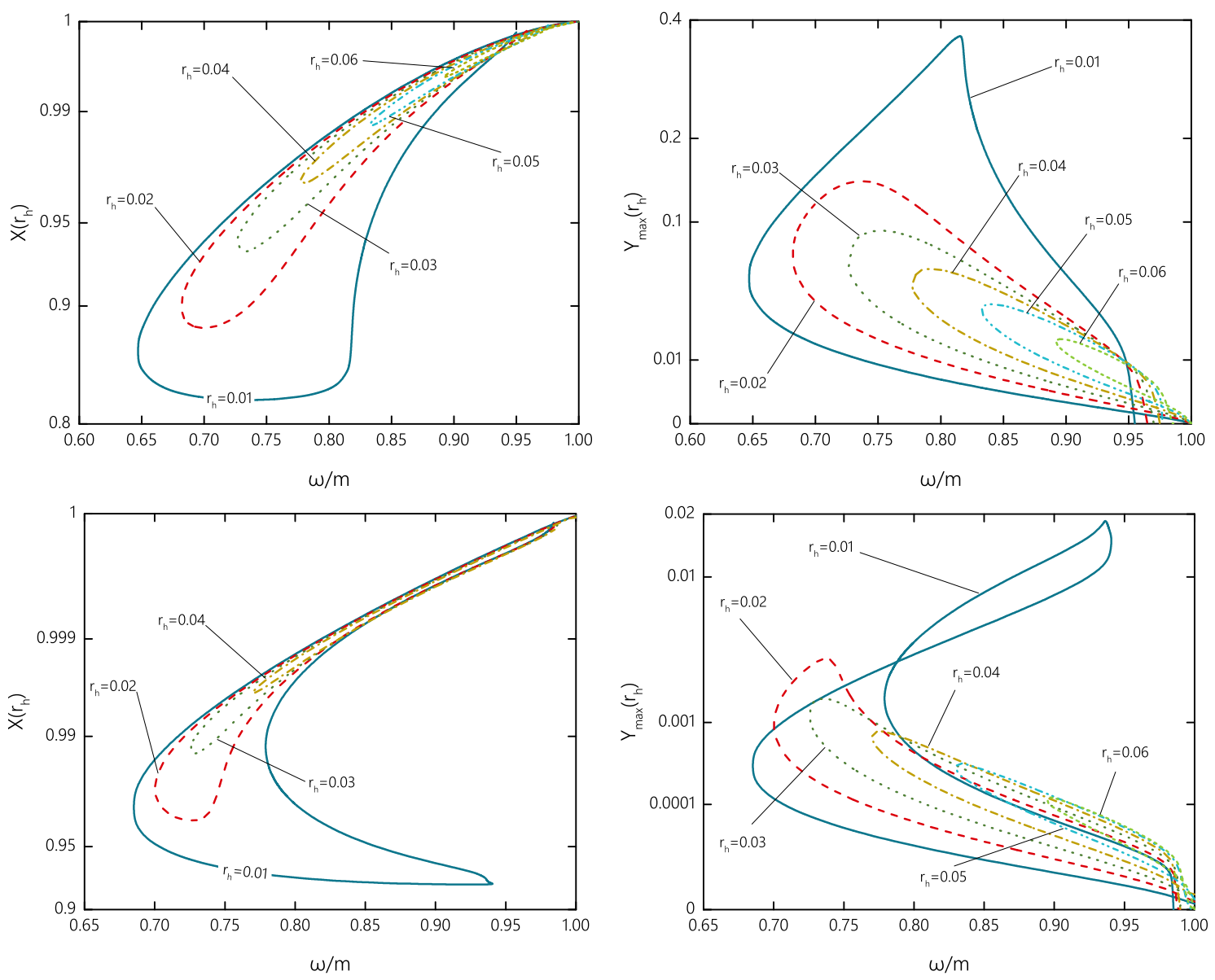

Figure 3. The value of real scalar field on the horizon $X\left(r_{h}\right)$ at $\theta=0$ (left column) and the maximal value of the complex scalar field on the horizon $Y_{\max }\left(r_{h}\right)$ (right column) vs the frequency $\omega$ for a set of values of the horizon radius parameter $r_{h}$ for $n=1$ rotating parity-even (upper row) and parity-odd (lower row) hairy black holes at $\mu / m=0.25$ and $\alpha=0.5$.

to the solutions with non-zero values of the mass parameter $\mu$ presented in figure 2 , the fundamental branch of the regular spinning boson stars arises in the limit $\omega_{\max }=1$, and the mass of the configuration increases as the frequency decreases. This branch extends up to some lower non-zero critical value $\omega_{\min }>0$, and the ADM mass of the boson star increases monotonically along this branch.

The lower critical value $\omega_{\text {min }}$ depends on the strength of the gravitational coupling, and increases slowly as $\alpha$ grows. In the flat space limit the axially-symmetric spinning Q-balls with massless real component exist over the entire range of values of the frequency $\omega \in[0,1][64,65]$. We constructed solutions with very large values of $\alpha$, and they are likely to exist for arbitrary values of the effective gravitational coupling.

This branch bifurcates with the second upper branch at $\omega_{\min }$, and we observe a small loop in the $M(\omega)$ dependence, as illustrated in figure 6 , left plot, see also the zoomed-in subplot in figure 7. This loop, which is observed both for the parity-even and parity-odd black holes with a synchronized massive component, disappears when the mass parameter $\mu$ increases sufficiently from zero. 


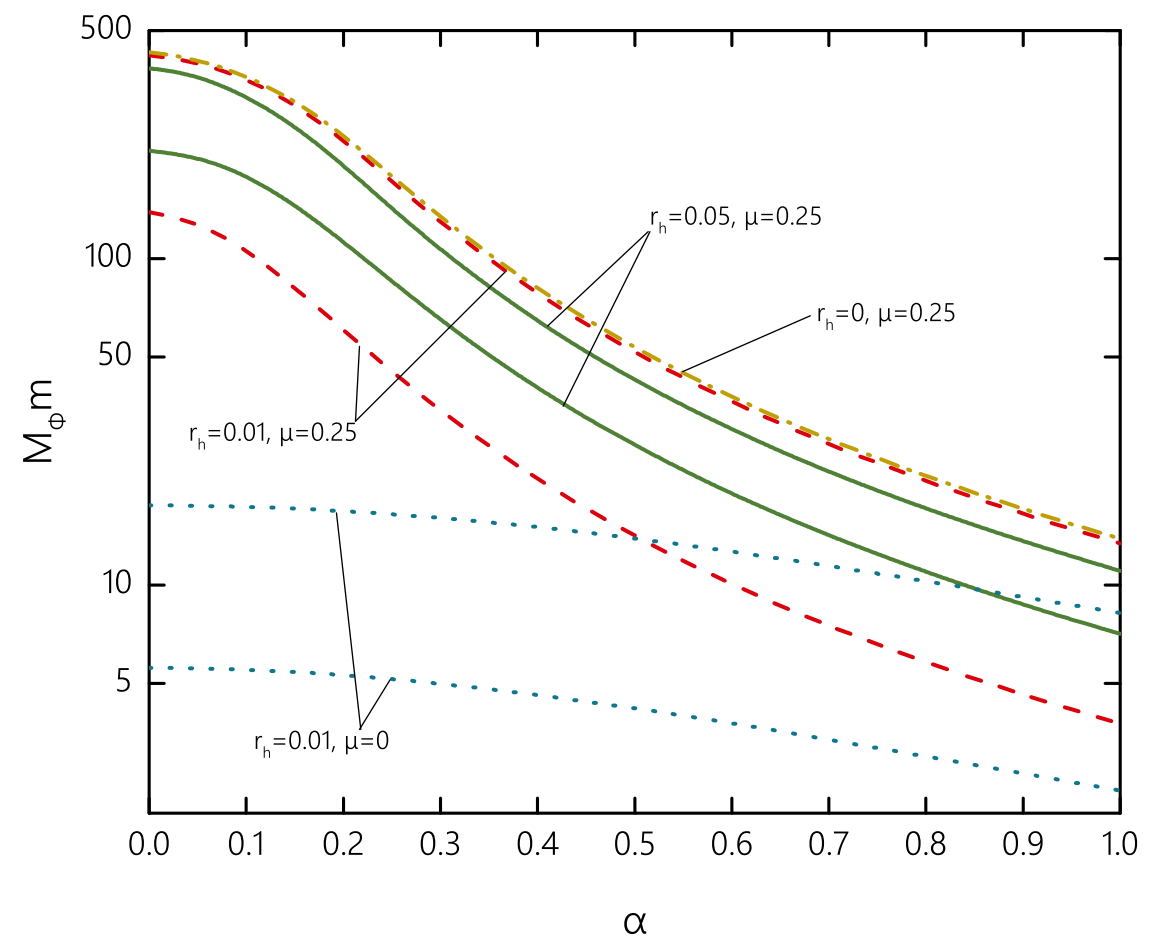

Figure 4. The scalar hair mass $M_{\Phi}$ vs the gravitational coupling constant $\alpha$ for a set of values of horizon radius parameter $r_{h}$ and the potential coupling constant $\mu$ for $n=1$ rotating parity-even hairy black holes at frequency $\omega / m=0.9$.

The mass of the configurations rapidly increases as the frequency $\omega$ approaches its minimal value $\omega_{\min }$. Further increase of the frequency $\omega$ along the second branch is related with a decrease of the mass. At some upper critical value of the frequency $\omega_{\mathrm{cr}}^{(2)}$ the curve backbends and a third (backward) branch of the regular spinning solitons is found. Thus, an overall inspiral type pattern is observed again for this sequence of solutions.

We have performed a similar study also for the parity-odd $\mu=0$ solutions with a longrange real component, see figure 7 . The branch structure of the spinning parity-odd black holes with synchronized hair is more explicit than the one of the corresponding parity-even solutions. In particular, for small values of the horizon radius, the third and the forth branch are clearly visible.

In addition, figure 8 exhibits the pattern of the evolution of the branches of the parityeven (upper row) and parity-odd (bottom row) solutions at the horizon radius $r_{h}=0.01$, as the mass parameter $\mu$ varies. We observe that the range of allowed values of the frequency rapidly decreases as $\mu$ grows. In the limiting case, $\mu \rightarrow \infty$, the real scalar component becomes trivial everywhere in space, so the model (2.1) becomes effectively truncated to the massive complex-Klein-Gordon theory minimally coupled to Einstein gravity [77].

It is known, that the latter model supports the existence of black holes with synchronised hair $[45,46,48]$. These solutions trivialize when the gravitational coupling is switched off. Indeed, our simulations show that the spinning component of the parity-even solutions of the model (2.1) approaches the corresponding solutions of the complex-Klein- 

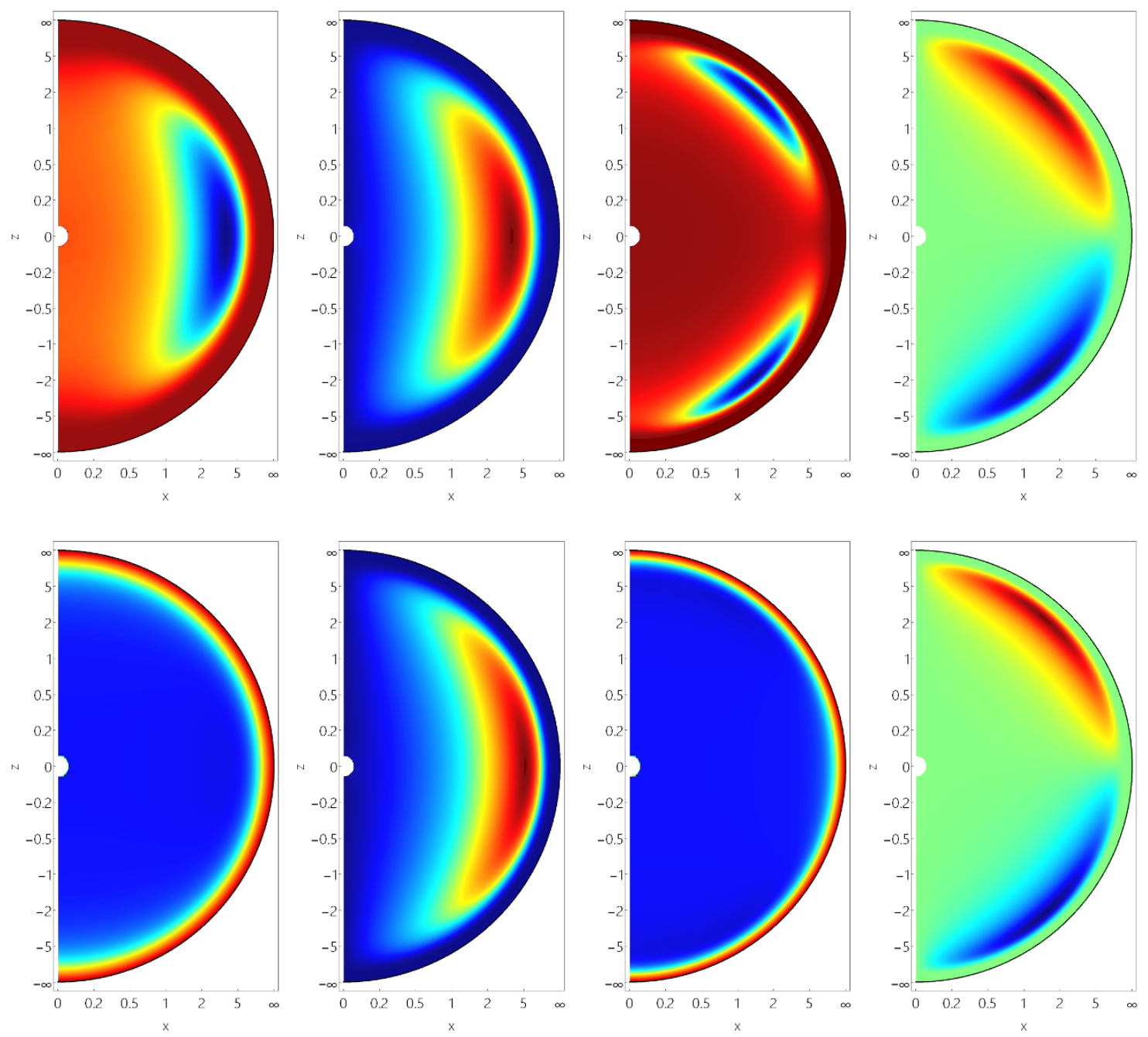

Figure 5. Contour plots of the profile function of the real scalar field $X$ (first and third columns) and the profile function of the complex scalar field $Y$ (second and fourth columns) in the $y=0$ plane for parity-even (left two columns) and parity-odd (right two columns) $n=1$ black holes with synchronized hair at $\omega / m=0.9, \alpha=0.5$ and horizon radius parameter $r_{h}=0.05$. The upper row shows solutions for a finite value of the real scalar field mass $\mu / m=0.25$, whilst the bottom row shows solutions for a massless real scalar field with $\mu=0$.

Gordon theory presented in $[46,48]$. Likewise, in the limit $\mu \rightarrow \infty$ the $n=1$ parity-odd solutions of the model (2.2) approach Kerr black holes with parity-odd synchronised scalar hair $[61,63]$.

Finally, we address the geometry of the ergo-surfaces of the hairy black holes in the Einstein-Friedberg-Lee-Sirlin model. The ergo-surfaces are defined as the zero locus of the normalized time-like Killing vector $\xi \cdot \xi=0$, or

$$
g_{t t}=-F_{0}+\sin ^{2} \theta F_{2} W^{2}=0 .
$$

We found that, in analogy to other known hairy black holes, there are both the ordinary 

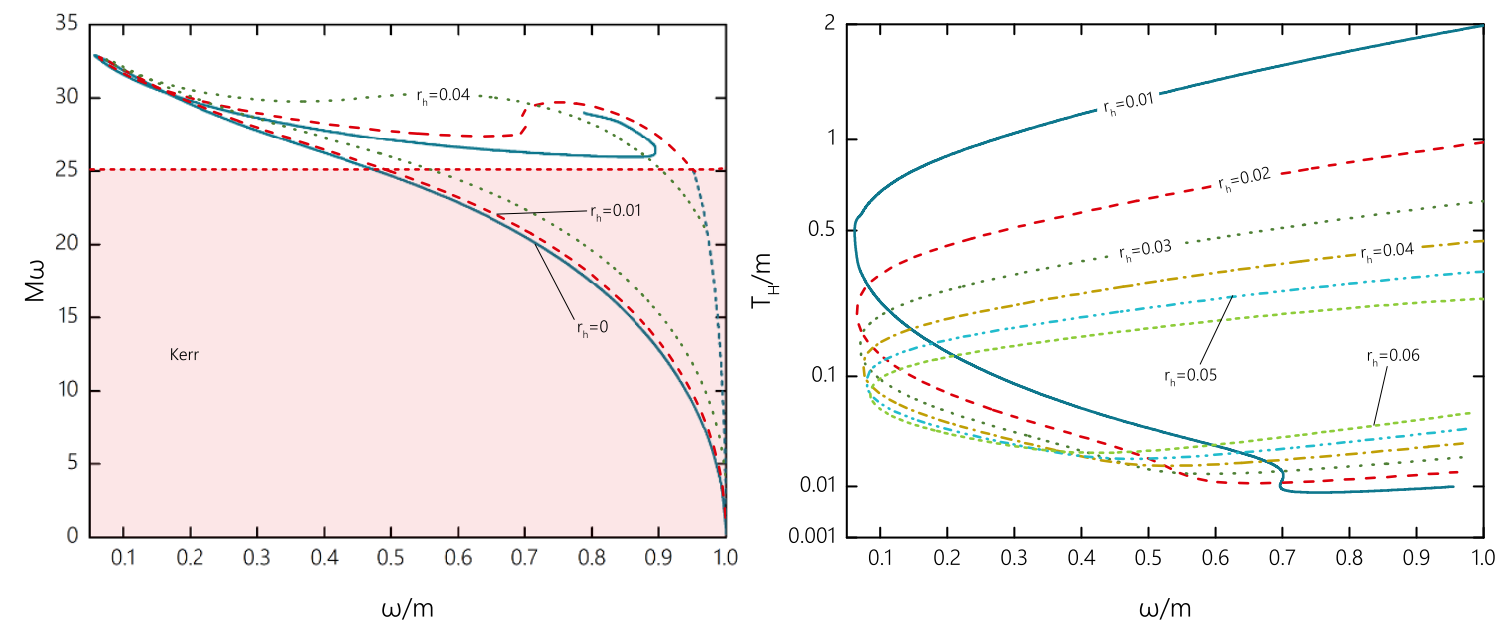

Figure 6. The ADM mass $M$ (left plot) and the Hawking temperature $T_{h}$ (right plot) vs the frequency $\omega$ for a set of values of the horizon radius parameter $r_{h}$ for $n=1$ rotating parity-even hairy black holes in the limiting case of vanishing scalar potential $(\mu=0)$ at $\alpha=0.5$. To simplify the presentation the ADM mass is multiplied by $\omega$.

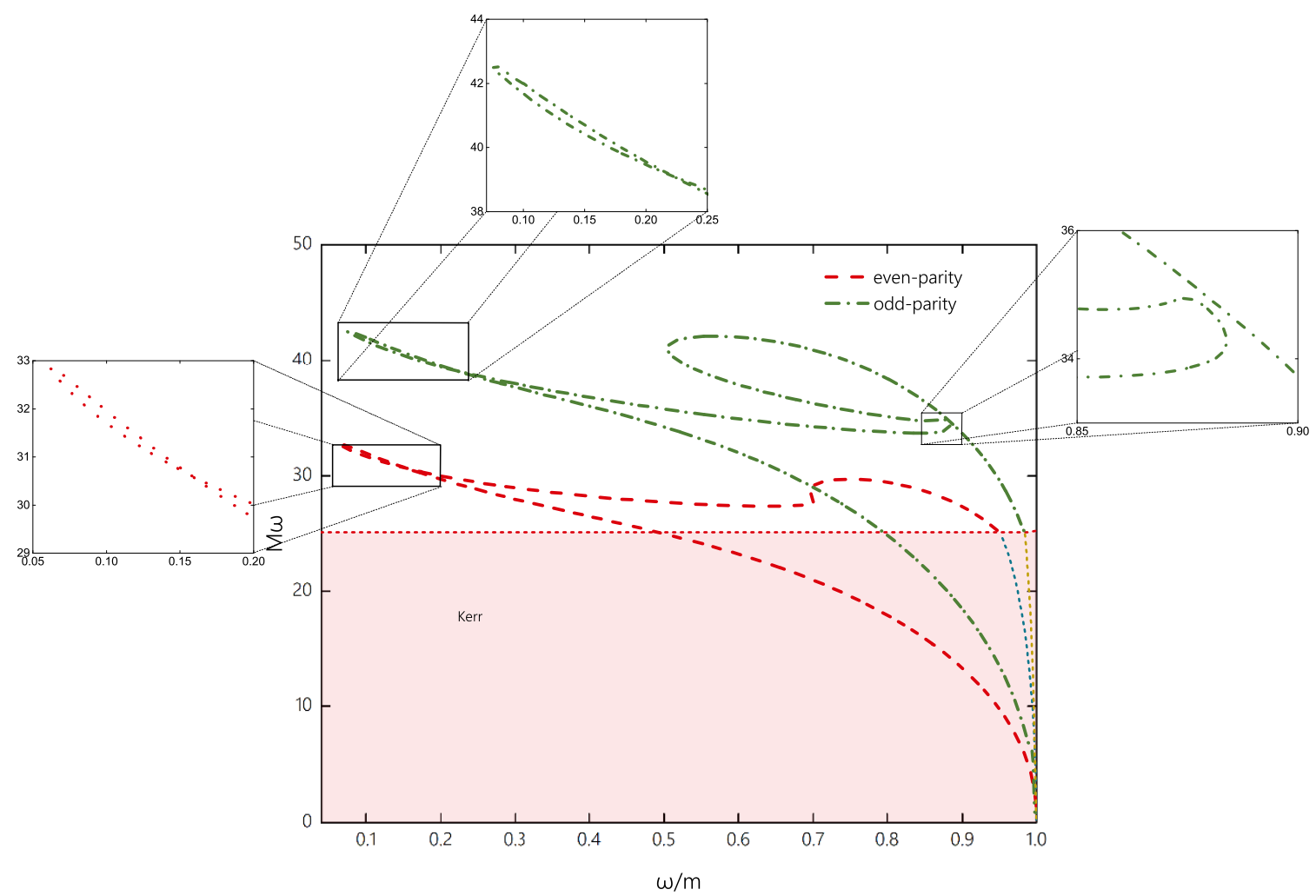

Figure 7. The ADM mass $M$ vs the frequency $\omega$ for parity-even and parity-odd $n=1$ rotating hairy black holes at $\mu=0, r_{h}=0.01$ and $\alpha=0.5$. To simplify the presentation the ADM mass is multiplied by $\omega$. 

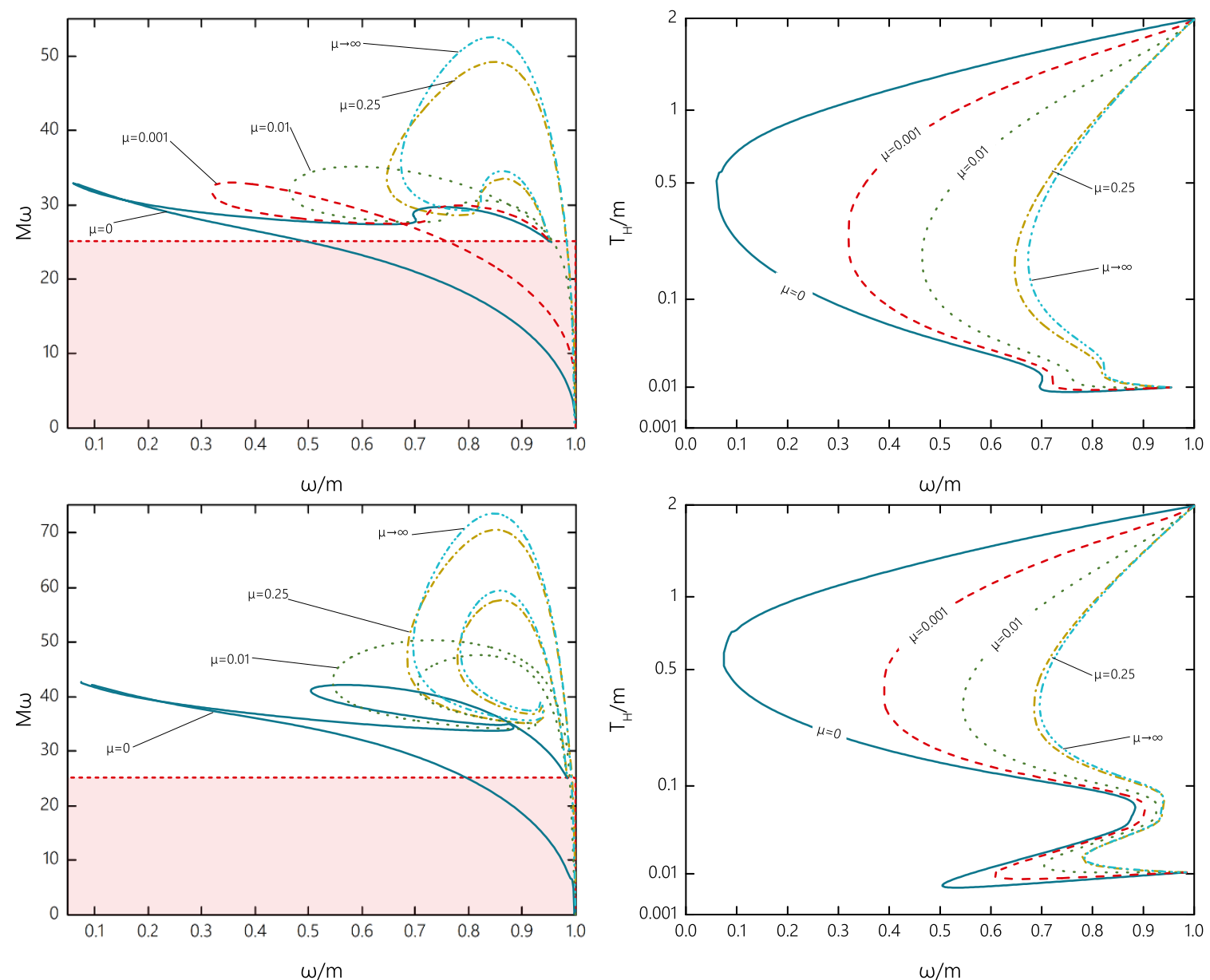

Figure 8. The ADM mass $M$ (left column) and the Hawking temperature $T_{h}$ (right column) vs the frequency $\omega$ for a set of values of the potential coupling constant $\mu / m$ for $n=1$ parity-even (upper row) and parity-odd (lower row) rotating hairy black holes at the horizon radius parameter $r_{h}=0.01$ and $\alpha=0.5$.

Kerr-like $S^{2}$ ergo-regions with topology $S^{2}$, which appear on the fundamental branch, and ergo-Saturns with topology $S^{2} \bigoplus\left(S^{1} \times S^{1}\right)$, when the black holes possess parity-even scalar hair $[47,53]$, see figure 9 . Further, analogously to the corresponding parity-odd solutions in the Einstein-Klein-Gordon theory [63], there is a new type of ergo-surfaces, which represent ergo-double-torus-Saturns with topology $\left(S^{1} \times S^{1}\right) \bigoplus\left(S^{1} \times S^{1}\right) \bigoplus S^{2}$, see figure 9 , right plot.

Also, we constructed spinning solutions for higher values of the azimuthal winding number $n>1$. Generally, these possess similar properties as the $n=1$ solutions, however, their mass is higher, and they exist in a larger interval of frequencies, as seen in figure 10.

\section{Conclusions}

In this work we have coupled the Friedberg-Lee-Sirlin model [11] to Einstein gravity and investigated some of its solutions. When the flat space non-topological solitons are coupled to gravity, regular boson star solutions arise. In the simplest case, these boson star 

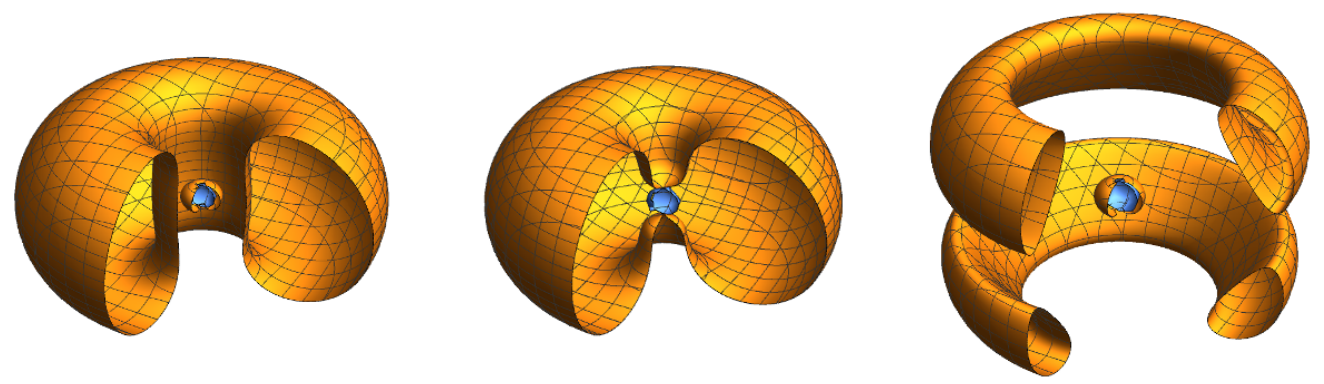

Figure 9. Ergosurfaces of $n=1$ parity-even hairy BHs with horizon radius parameter $r_{h}=0.01$ and frequencies $\omega / m=0.35(1), \omega / m=0.5(2)$ and parity-odd BH with $\omega / m=0.5$ (3) on the backward branch. Blue surfaces represent the horizon.
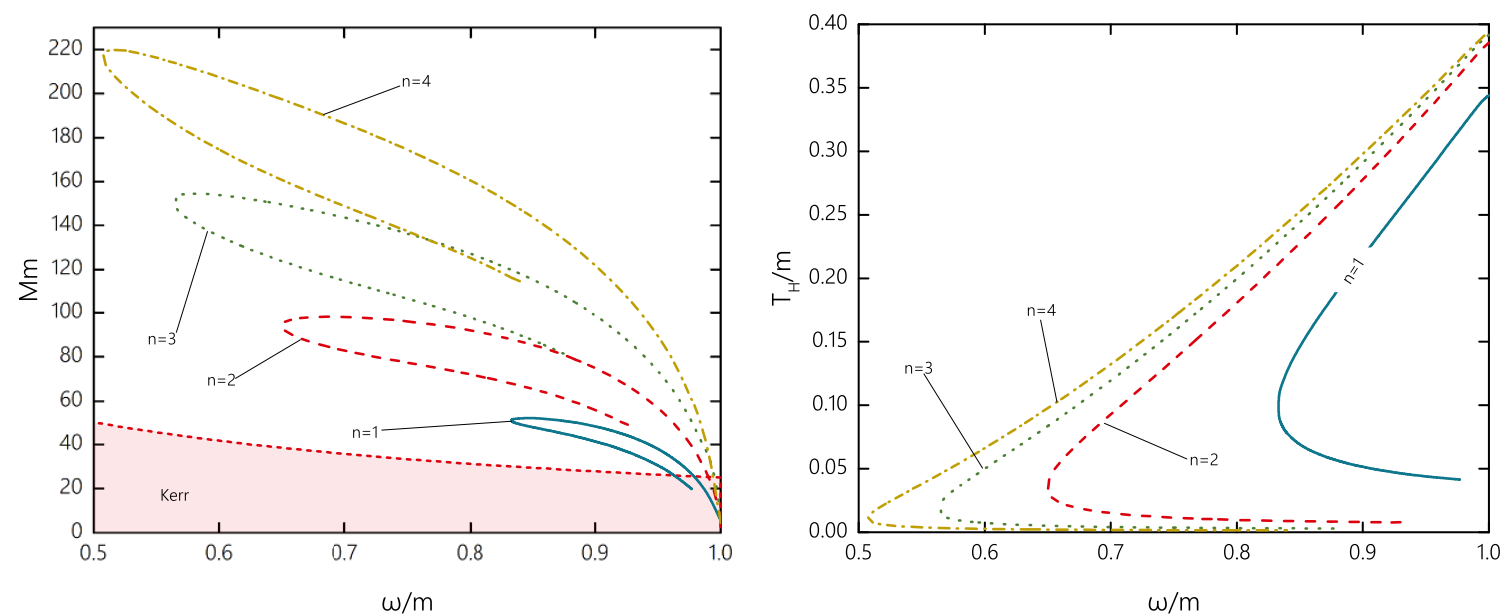

Figure 10. The ADM mass $M$ (left plot) and the Hawking temperature $T_{h}$ (right plot) vs the frequency $\omega / m$ for a set of values of the winding number $n$ for rotating parity-even hairy black holes at the horizon radius $r_{h}=0.05$ and $\mu / m=0.25$.

solutions are spherically symmetric. Here, however, we have concentrated on their rotating generalizations, which may feature a parity-even or parity-odd complex scalar field.

The properties of these boson stars are similar to those found in models with a complex scalar field only, like the Einstein-Klein-Gordon model. In fact the solutions of this model are recovered, when the coupling constant $\mu$ is taken to infinity. On the other hand, also boson star solutions with a massless real scalar field have been found.

When the presence of an event horizon together with the synchronization condition between the frequency and the horizon angular velocity is imposed, new hairy black holes emerge in a certain region of the parameter space. According to the symmetries of the complex scalar field they represent parity-even or parity-odd hairy black holes.

These hairy black holes possess two types of hair, consisting of the usual complex scalar field hair and the additional real scalar field hair. In particular, when the mass of the real scalar field vanishes, the real scalar field hair becomes long-ranged, whereas the complex scalar field hair remains massive. This constitutes an interesting new quality of hairy black holes. 
We note that similar hairy black hole solutions as the ones studied here may also exist in the Wick-Cutkosky model [72, 73], recently revisited in [74, 75], if the corresponding Q-ball configurations are coupled to Einstein gravity.

Various interesting features of the regular and hairy black hole solutions of the EinsteinFriedberg-Lee-Sirlin model remain to be studied, and, in particular, there should be numerous further radially and angularly excited regular and hairy black hole solutions in the model.

\section{Acknowledgments}

We are grateful to Burkhard Kleihaus and Eugen Radu for inspiring and valuable discussions. This work was supported in part by the DFG Research Training Group 1620 Models of Gravity as well as by the COST Action CA16104 GWverse. Ya.S. gratefully acknowledges the support of the Alexander von Humboldt Foundation and the partial support of the Ministry of Science and High Education of Russian Federation, project No. 3.1386.2017. I.P. would like to acknowledge support by the DAAD Ostpartnerschaft Programm.

Open Access. This article is distributed under the terms of the Creative Commons Attribution License (CC-BY 4.0), which permits any use, distribution and reproduction in any medium, provided the original author(s) and source are credited.

\section{References}

[1] D.J. Kaup, Klein-Gordon Geon, Phys. Rev. 172 (1968) 1331 [InSPIRE].

[2] R. Ruffini and S. Bonazzola, Systems of selfgravitating particles in general relativity and the concept of an equation of state, Phys. Rev. 187 (1969) 1767 [InSPIRE].

[3] H. Lückock and I. Moss, Black Holes Have Skyrmion Hair, Phys. Lett. B 176 (1986) 341 [INSPIRE].

[4] S. Droz, M. Heusler and N. Straumann, New black hole solutions with hair, Phys. Lett. B 268 (1991) 371 [INSPIRE].

[5] P. Bizon and T. Chmaj, Gravitating skyrmions, Phys. Lett. B 297 (1992) 55 [InSPIRE].

[6] R. Bartnik and J. Mckinnon, Particle-Like Solutions of the Einstein Yang-Mills Equations, Phys. Rev. Lett. 61 (1988) 141 [INSPIRE].

[7] K.-M. Lee, V.P. Nair and E.J. Weinberg, Black holes in magnetic monopoles, Phys. Rev. D 45 (1992) 2751 [hep-th/9112008] [INSPIRE].

[8] P. Breitenlohner, P. Forgacs and D. Maison, Gravitating monopole solutions, Nucl. Phys. B 383 (1992) 357 [INSPIRE].

[9] B.R. Greene, S.D. Mathur and C.M. O'Neill, Eluding the no hair conjecture: Black holes in spontaneously broken gauge theories, Phys. Rev. D 47 (1993) 2242 [hep-th/9211007] [INSPIRE].

[10] P. Breitenlohner, P. Forgacs and D. Maison, Gravitating monopole solutions. 2, Nucl. Phys. B 442 (1995) 126 [gr-qc/9412039] [InSPIRE]. 
[11] R. Friedberg, T.D. Lee and A. Sirlin, A Class of Scalar-Field Soliton Solutions in Three Space Dimensions, Phys. Rev. D 13 (1976) 2739 [inSPIRE].

[12] S.R. Coleman, Q Balls, Nucl. Phys. B 262 (1985) 263 [Erratum ibid. B 269 (1986) 744] [INSPIRE].

[13] G. 't Hooft, Magnetic Monopoles in Unified Gauge Theories, Nucl. Phys. B 79 (1974) 276 [INSPIRE].

[14] A.M. Polyakov, Particle Spectrum in the Quantum Field Theory, JETP Lett. 20 (1974) 194 [INSPIRE].

[15] F.R. Klinkhamer and N.S. Manton, A Saddle Point Solution in the Weinberg-Salam Theory, Phys. Rev. D 30 (1984) 2212 [INSPIRE].

[16] T.H.R. Skyrme, A Nonlinear field theory, Proc. Roy. Soc. Lond. A 260 (1961) 127 [inSPIRE].

[17] R. Friedberg, T.D. Lee and Y. Pang, Mini-soliton stars, Phys. Rev. D 35 (1987) 3640 [INSPIRE].

[18] R. Friedberg, T.D. Lee and Y. Pang, Scalar Soliton Stars and Black Holes, Phys. Rev. D 35 (1987) 3658 [INSPIRE].

[19] B. Kleihaus, J. Kunz and M. List, Rotating boson stars and Q-balls, Phys. Rev. D 72 (2005) 064002 [gr-qc/0505143] [inSPIRE].

[20] B. Kleihaus, J. Kunz, M. List and I. Schaffer, Rotating Boson Stars and Q-Balls. II. Negative Parity and Ergoregions, Phys. Rev. D 77 (2008) 064025 [arXiv:0712.3742] [INSPIRE].

[21] M.S. Volkov and D.V. Galtsov, NonAbelian Einstein Yang-Mills black holes, JETP Lett. 50 (1989) 346 [INSPIRE].

[22] R. Ruffini and J.A. Wheeler, Introducing the black hole, Phys. Today 24 (1971) 30.

[23] M.S. Volkov and D.V. Gal'tsov, Gravitating nonAbelian solitons and black holes with Yang-Mills fields, Phys. Rept. 319 (1999) 1 [hep-th/9810070] [INSPIRE].

[24] C.A.R. Herdeiro and E. Radu, Asymptotically flat black holes with scalar hair: a review, Int. J. Mod. Phys. D 24 (2015) 1542014 [arXiv:1504.08209] [INSPIRE].

[25] M.S. Volkov, Hairy black holes in the XX-th and XXI-st centuries, in Proceedings, 14 th Marcel Grossmann Meeting on Recent Developments in Theoretical and Experimental General Relativity, Astrophysics and Relativistic Field Theories (MG14) (In 4 Volumes): Rome, Italy, July 12-18, 2015, vol. 2, pp. 1779-1798, 2017, arXiv:1601.08230 [INSPIRE].

[26] I. Pena and D. Sudarsky, Do collapsed boson stars result in new types of black holes?, Class. Quant. Grav. 14 (1997) 3131 [INSPIRE].

[27] S. Hod, No-go theorem for static boson stars, Phys. Lett. B 778 (2018) 239 [arXiv: 1902.05230] [INSPIRE].

[28] C. Adam, O. Kichakova, Ya. Shnir and A. Wereszczynski, Hairy black holes in the general Skyrme model, Phys. Rev. D 94 (2016) 024060 [arXiv: 1605.07625] [INSPIRE].

[29] S.B. Gudnason, M. Nitta and N. Sawado, Black hole Skyrmion in a generalized Skyrme model, JHEP 09 (2016) 055 [arXiv: 1605.07954] [INSPIRE].

[30] I. Perapechka and Y. Shnir, Generalized Skyrmions and hairy black holes in asymptotically $A d S_{4}$ spacetime, Phys. Rev. D 95 (2017) 025024 [arXiv:1612.01914] [INSPIRE]. 
[31] Y. Kobayashi, M. Kasai and T. Futamase, Does a boson star rotate?, Phys. Rev. D 50 (1994) 7721 [INSPIRE].

[32] F.E. Schunck and E.W. Mielke, Rotating boson star as an effective mass torus in general relativity, Phys. Lett. A 249 (1998) 389 [INSPIRE].

[33] F.D. Ryan, Spinning boson stars with large selfinteraction, Phys. Rev. D 55 (1997) 6081 [INSPIRE].

[34] S. Yoshida and Y. Eriguchi, Rotating boson stars in general relativity, Phys. Rev. D 56 (1997) 762 [INSPIRE].

[35] T. Ioannidou, B. Kleihaus and J. Kunz, Spinning gravitating skyrmions, Phys. Lett. B 643 (2006) 213 [gr-qc/0608110] [INSPIRE].

[36] B. Kleihaus, J. Kunz and U. Neemann, Gravitating stationary dyons and rotating vortex rings, Phys. Lett. B 623 (2005) 171 [gr-qc/0507047] [INSPIRE].

[37] B. Kleihaus, J. Kunz, F. Navarro-Lerida and U. Neemann, Stationary Dyonic Regular and Black Hole Solutions, Gen. Rel. Grav. 40 (2008) 1279 [arXiv:0705.1511] [InSPIRE].

[38] C. Herdeiro, I. Perapechka, E. Radu and Ya. Shnir, Gravitating solitons and black holes with synchronised hair in the four dimensional O(3) $\sigma$-model, JHEP 02 (2019) 111 [arXiv: 1811.11799] [INSPIRE].

[39] B. Kleihaus and J. Kunz, Rotating hairy black holes, Phys. Rev. Lett. 86 (2001) 3704 [gr-qc/0012081] [INSPIRE].

[40] B. Kleihaus, J. Kunz and F. Navarro-Lerida, Rotating Einstein-Yang-Mills black holes, Phys. Rev. D 66 (2002) 104001 [gr-qc/0207042] [InSPIRE].

[41] B. Kleihaus, J. Kunz and F. Navarro-Lerida, Rotating dilaton black holes with hair, Phys. Rev. D 69 (2004) 064028 [gr-qc/0306058] [INSPIRE].

[42] B. Kleihaus, J. Kunz and F. Navarro-Lerida, Stationary black holes with static and counter rotating horizons, Phys. Rev. D 69 (2004) 081501 [gr-qc/0309082] [INSPIRE].

[43] B. Kleihaus, J. Kunz and F. Navarro-Lerida, Rotating black holes with monopole hair, Phys. Lett. B 599 (2004) 294 [gr-qc/0406094] [INSPIRE].

[44] B. Kleihaus, J. Kunz and F. Navarro-Lerida, Rotating black holes with non-Abelian hair, Class. Quant. Grav. 33 (2016) 234002 [arXiv:1609.07357] [INSPIRE].

[45] S. Hod, Stationary Scalar Clouds Around Rotating Black Holes, Phys. Rev. D 86 (2012) 104026 [Erratum ibid. D 86 (2012) 129902] [arXiv:1211.3202] [InSPIRE].

[46] C.A.R. Herdeiro and E. Radu, Kerr black holes with scalar hair, Phys. Rev. Lett. 112 (2014) 221101 [arXiv: 1403.2757] [INSPIRE].

[47] C. Herdeiro and E. Radu, Ergosurfaces for Kerr black holes with scalar hair, Phys. Rev. D 89 (2014) 124018 [arXiv: 1406.1225] [InSPIRE].

[48] C. Herdeiro and E. Radu, Construction and physical properties of Kerr black holes with scalar hair, Class. Quant. Grav. 32 (2015) 144001 [arXiv:1501.04319] [INSPIRE].

[49] S. Hod, Kerr-Newman black holes with stationary charged scalar clouds, Phys. Rev. D 90 (2014) 024051 [arXiv: 1406.1179] [INSPIRE].

[50] C.L. Benone, L.C.B. Crispino, C. Herdeiro and E. Radu, Kerr-Newman scalar clouds, Phys. Rev. D 90 (2014) 104024 [arXiv:1409.1593] [INSPIRE]. 
[51] C. Herdeiro, E. Radu and H. Rúnarsson, Non-linear Q-clouds around Kerr black holes, Phys. Lett. B 739 (2014) 302 [arXiv: 1409.2877] [INSPIRE].

[52] C.A.R. Herdeiro and E. Radu, A new spin on black hole hair, Int. J. Mod. Phys. D 23 (2014) 1442014 [arXiv:1405.3696] [INSPIRE].

[53] B. Kleihaus, J. Kunz and S. Yazadjiev, Scalarized Hairy Black Holes, Phys. Lett. B 744 (2015) 406 [arXiv: 1503.01672] [INSPIRE].

[54] C.A.R. Herdeiro, E. Radu and H. Rúnarsson, Kerr black holes with self-interacting scalar hair: hairier but not heavier, Phys. Rev. D 92 (2015) 084059 [arXiv:1509.02923] [InSPIRE].

[55] C. Herdeiro, J. Kunz, E. Radu and B. Subagyo, Myers-Perry black holes with scalar hair and a mass gap: Unequal spins, Phys. Lett. B 748 (2015) 30 [arXiv:1505.02407] [INSPIRE].

[56] C. Herdeiro, E. Radu and H. Rúnarsson, Kerr black holes with Proca hair, Class. Quant. Grav. 33 (2016) 154001 [arXiv: 1603.02687] [INSPIRE].

[57] Y. Brihaye, C. Herdeiro and E. Radu, Inside black holes with synchronized hair, Phys. Lett. B 760 (2016) 279 [arXiv: 1605.08901] [InSPIRE].

[58] S. Hod, Extremal Kerr-Newman black holes with extremely short charged scalar hair, Phys. Lett. B 751 (2015) 177 [arXiv:1707.06246] [INSPIRE].

[59] C. Herdeiro, J. Kunz, E. Radu and B. Subagyo, Probing the universality of synchronised hair around rotating black holes with Q-clouds, Phys. Lett. B 779 (2018) 151 [arXiv:1712.04286] [INSPIRE].

[60] C. Herdeiro, I. Perapechka, E. Radu and Ya. Shnir, Skyrmions around Kerr black holes and spinning BHs with Skyrme hair, JHEP 10 (2018) 119 [arXiv:1808.05388] [INSPIRE].

[61] Y.-Q. Wang, Y.-X. Liu and S.-W. Wei, Excited Kerr black holes with scalar hair, Phys. Rev. D 99 (2019) 064036 [arXiv: 1811.08795] [INSPIRE].

[62] J.F.M. Delgado, C.A.R. Herdeiro and E. Radu, Kerr black holes with synchronised scalar hair and higher azimuthal harmonic index, Phys. Lett. B 792 (2019) 436 [arXiv: 1903.01488] [INSPIRE].

[63] J. Kunz, I. Perapechka and Ya. Shnir, Kerr black holes with parity-odd scalar hair, arXiv: 1904.07630 [INSPIRE].

[64] A. Levin and V. Rubakov, Q-balls with scalar charges, Mod. Phys. Lett. A 26 (2011) 409 [arXiv: 1010.0030] [INSPIRE].

[65] V. Loiko, I. Perapechka and Ya. Shnir, Q-balls without a potential, Phys. Rev. D 98 (2018) 045018 [arXiv: 1805.11929 ] [INSPIRE].

[66] M.S. Volkov and E. Wohnert, Spinning Q balls, Phys. Rev. D 66 (2002) 085003 [hep-th/0205157] [INSPIRE].

[67] E. Radu and M.S. Volkov, Existence of stationary, non-radiating ring solitons in field theory: knots and vortons, Phys. Rept. 468 (2008) 101 [arXiv:0804.1357] [INSPIRE].

[68] Y. Brihaye and B. Hartmann, Angularly excited and interacting boson stars and Q-balls, Phys. Rev. D 79 (2009) 064013 [arXiv:0812.3968] [INSPIRE].

[69] T. Tamaki and N. Sakai, Unified picture of Q-balls and boson stars via catastrophe theory, Phys. Rev. D 81 (2010) 124041 [arXiv:1105.1498] [INSPIRE]. 
[70] L.G. Collodel, B. Kleihaus and J. Kunz, Excited Boson Stars, Phys. Rev. D 96 (2017) 084066 [arXiv: 1708.02057] [INSPIRE].

[71] I. Perapechka and Y. Shnir, Spinning gravitating Skyrmions in a generalized Einstein-Skyrme model, Phys. Rev. D 96 (2017) 125006 [arXiv:1710.06334] [INSPIRE].

[72] G.C. Wick, Properties of Bethe-Salpeter Wave Functions, Phys. Rev. 96 (1954) 1124 [INSPIRE].

[73] R.E. Cutkosky, Solutions of a Bethe-Salpeter equations, Phys. Rev. 96 (1954) 1135 [InSPIRE].

[74] E. Ya. Nugaev and M.N. Smolyakov, Q-balls in the Wick-Cutkosky model, Eur. Phys. J. C 77 (2017) 118 [arXiv: 1605.02056] [INSPIRE].

[75] A.G. Panin and M.N. Smolyakov, Classical behaviour of Q-balls in the Wick-Cutkosky model, Eur. Phys. J. C 79 (2019) 150 [arXiv:1810.03558] [INSPIRE].

[76] J. Kunz, E. Radu and B. Subagyo, Gravitating vortons as ring solitons in general relativity, Phys. Rev. D 87 (2013) 104022 [arXiv:1303.1003] [INSPIRE].

[77] F.E. Schunck and E.W. Mielke, General relativistic boson stars, Class. Quant. Grav. 20 (2003) R301 [arXiv:0801.0307] [INSPIRE].

[78] T.D. Lee and Y. Pang, Stability of Mini-Boson Stars, Nucl. Phys. B 315 (1989) 477 [INSPIRE].

[79] F.V. Kusmartsev, E.W. Mielke and F.E. Schunck, Gravitational stability of boson stars, Phys. Rev. D 43 (1991) 3895 [arXiv:0810.0696] [InSPIRE].

[80] B. Kleihaus, J. Kunz and S. Schneider, Stable Phases of Boson Stars, Phys. Rev. D 85 (2012) 024045 [arXiv: 1109.5858] [INSPIRE]. 